\section{AB0263 ASSOCIATED FACTORS OF RESIDUAL SYMPTOMS AMONG PATIENTS WITH RHEUMATOID ARTHRITIS IN REMISSION OR LOW DISEASE ACTIVITY}

Takeshi Kashiwagura $^{1}$, Moto Kobayashi ${ }^{2}$, Masakazu Urayama ${ }^{3}$, Natsuo Konishi ${ }^{1}$, Toshiaki Aizawa $^{4}$, Hiroki Itoh ${ }^{5}$, Yusuke Sugimura ${ }^{1}$, Tetsuya Kawano ${ }^{4,6}$, Hiroshi Aonuma ${ }^{3}$, Tsutomu Sakuraba ${ }^{2}$, Takayuki Tani $^{7}$, Hidekazu Abe $^{8}$ Norio Suzuki ${ }^{9}$, Keiji Kamo $^{10}$, Yosuke Iwamoto ${ }^{1}$, Takanori Miura ${ }^{7}$, Yoshiaki Kimura ${ }^{1}$ Naohisa Miyakoshi', Yoichi Shimada'. ' ${ }^{1}$ Akita City Hospital, Department of Orthopedic Surgery, Akita, Japan; ${ }^{2}$ Hiraka General Hospital, Yokote, Japan; ${ }^{3}$ Ogachi Central Hospital, Yuzawa, Japan; ${ }^{4}$ Kita Akita Municipal Hospital, Kita Akita, Japan; ${ }^{5}$ Noshiro Kousei Medical Center, Noshiro, Japan; ${ }^{6}$ Akita University Graduate School of Medicine, Akita, Japan; ${ }^{7}$ Kakunodate Municipal Hospital, Senboku, Japan; ${ }^{8}$ Ugo Municipal Hospital, Ugo, Japan; ${ }^{9}$ Yuri Kumiai General Hospital, Yuri Honjo, Japan; ${ }^{10}$ Akita Rosai Hospital, Odate, Japan

Background: Treatment outcomes for rheumatoid arthritis (RA) have been improved by advances in drugs. In daily clinical practice, treatment outcomes are assessed with both inflammatory findings and based on swollen joints (SJ), tender joints (TJ), a patient's global assessment on visual analogue scale (GVAS) score, a physician's GVAS score, etc. Although composite measures indicate suppressed inflammatory or articular symptoms, many patients show no improvement in GVAS scores. The reported residual symptoms include morning stiffness (MS), pain $(\mathrm{P})$, and dullness (D).

Objectives: We investigated the residual symptoms of patients achieving low disease activity (LDA) or complete remission (CR).

Methods: His study included 111 RA patients who received outpatient treatment at our department ( 31 men and 80 women). The mean age was 65.1 (range, 27-89) years. The disease stages were distributed as follows: 36 patients with Stage 1, 23 patients with Stage 2, 20 patients with Stage 3, and 32 patients with Stage 4 . The severity of dysfunction was distributed as follows: Class 1 in 73 patients, Class 2 in 25 patients, Class 3 in 11 patients, and Class 4 in 2 patients (Steinbrocker classification). Based on the disease activity score in 28 joints using the erythrocyte sedimentation rate, disease activity was graded as CR in 54 patients, LDA in 19, moderate disease activity in 37 , and high disease activity in 1. Questionnaire forms were used to assess the presence or absence and duration of MS and the presence or absence and severity of $P$ (Pain VAS) and of $D$ (Dullness VAS). These variables were assessed to determine how they were associated with clinical outcome measures, drugs, and surgery.

Results: The following residual symptoms were observed in 73 patients who achieved the treatment target of LDA or CR: MS in 34 patients $(46.6 \%), P$ in $48(65.6 \%)$, and $\mathrm{D}$ in $38(52.8 \%)$. In the LDA+CR group, the Pain VAS $(P<0.001, r=0.612)$ and Dullness VAS $(P=0.0131, r=$ 0.193 ) were significantly correlated with the GVAS. In patients with $P$ in the LDA+CR group, the use rates for analgesics $(P=0.0047)$ and biological disease-modifying antirheumatic drugs (bDMARDs) $(P=0.0295)$ were significantly higher. In the LDA+CR group, Dullness VAS scores were significantly improved by the use of bDMARDs $(P=0.0074)$. No association was observed between surgical interventions (42 patients) and residual symptoms. Conclusion: Residual symptoms were frequently observed even in patients achieving LDA or CR, which is regarded as the treatment target. Because $P$ and $D$ were significantly correlated with the GVAS, relief of these residual symptoms is expected to improve treatment effects. Although $D$ was relieved by bDMARDs, further studies on the treatment of residual $\mathrm{P}$ in patients achieving LDA or CR are necessary.

Disclosure of Interests: None declared

DOI: 10.1136/annrheumdis-2019-eular.4049

\section{AB0264 THE EXTENT OF JOINT DESTRUCTION IN THE KNEE JOINT AT THE INITIATION OF BDMARDS TREATMENT IS A PREDICTIVE FACTOR OF SUBSEQUENT TOTAL KNEE ARTHROPLASTY AND PROGRESSION OF JOINT DESTRUCTION IN PATIENTS WITH RHEUMATOID ARTHRITIS DURING LONG-TERM BDMARDS TREATMENT}

Daisuke Kihira, Yuji Hirano, Yukiyoshi Oishi. Toyohashi Municipal Hospital, Rheumatology, Toyohashi, Japan

Background: The joint destruction inhibitory effect of biological diseasemodifying anti-rheumatic drugs (bDMARDs) in patients with rheumatoid arthritis (RA) has been reported in a lot of studies. Most of them are evaluated in small joints such as hands and feet, there are few reports on the joint destruction inhibitory effect in large joints such as knees which are frequently affected in disease course of $R A^{11}$. Although bDMARDs is very effective drugs, we experience RA patients in whom total knee arthroplasties (TKA) are performed after the initiation of bDMARDs treatment ${ }^{2}$.
Objectives: To investigate the rate of TKA during bDMARDs treatment and to explore associated factors of subsequent TKA after the initiation of bDMARDs treatment in RA patients.

Methods: 356 knee joints of 184 RA patients who initiated bDMARDs treatment in our institute from March

2004 to November 2013 were included in this retrospective study. They were retrospectively studied over the course of a minimum of 5 years of follow up. Knees in which TKA were already performed before the initiation of bDMARDs treatment were excluded in this study. Patients' characteristics and rates of subsequent TKA over time were investigated. Next, factors associated with subsequent TKA were explored using univariate and multivariate analysis. The X-ray change (Larsen grade ${ }^{3)}$, ARASH score ${ }^{4)}$ ) at the last observation after 5 years or more was also examined. ARASHI score was developed in Japan to assess destruction of large joints such as knees or hips.

Results: Baseline patients' characteristics were below. Mean age was 57.1 years old. 152 female and 32 male. MTX was concomitant in $85.7 \%$ of patients. Prednisolone was concomitant in $53.7 \%$ of patients, First bDMARDs were infliximab in 67 cases, etanercept in 67 cases, adalimumab in 35 cases, tocilizumab in 6 cases, abatacept in 9 cases, golimumab in 0 cases and certolizumab in 0 cases. Agents were changed according to clinica necessity. The median follow-up period was 7.8 years. TKA were performed in 20 knees $(5.95 \%)$ in total. Baseline extent of joint destruction of knees which was evaluated using Larsen grade and ARASHI score, joint destruction, age, disease duration, steinbrocker class, concomitant corticosteroids, concomitant methotrexate, DAS28-CRP, SDAI, MMP-3, mHAQ, swelling of the knees,and tenderness of the knees were associated with subsequent TKA in univariates analysis. Multivariate analysis revealed that the extent of baseline joint Destruction and $\mathrm{mHAQ}$ were associated with subsequent TKA (Larsen grade: Odds ratio; 6.17, 95\%, confidence interval; 2.93-12.98, ARASHI score: Odds ratio; 3.50, 95\% confidence interval; 2.18-5.62, mHAQ Odds ratio; 3.56, 95\% confidence interval; 1.06-11.93). Cut-off value was calculated as 2 in Larsen grade, 3 in ARASHI score and 1.5 in mHAQ using receiver operating characteristic analysis. There were significant differences in the rates of subsequent TKA if knees were divided according to baseline Larsen grade, ARASHI score and mHAQ (Fig1). Baseline ARASHI status score in progressed knees which was worsened after over 5 years were increased compared with that in knees which was stable. There was no significant difference in baseline Larsen grade between progressed knees and non-progressed knees.

Conclusion: It was suggested that baseline joint destruction and $\mathrm{mHAQ}$ at the initiation of bDMARDs in RA patients was predictive factors of subsequent TKA and progression of joint destruction of knees during long-term bDMARDs treatment. Early intervention with effective bDMARDs is necessary to prevent destruction of knee joints and subsequent TKA in RA patients whose knee joints are affected.

\section{REFERENCES}

[1] Drossaers-Bakker, et al. Rheumatology. 2000

[2] Seki, et al. Clin Rheumatol. 2009

[3] Larsen, et al. Scand J Rheumatol. 1979

[4] Kaneko, et al. Mod Rheumatol. 2013

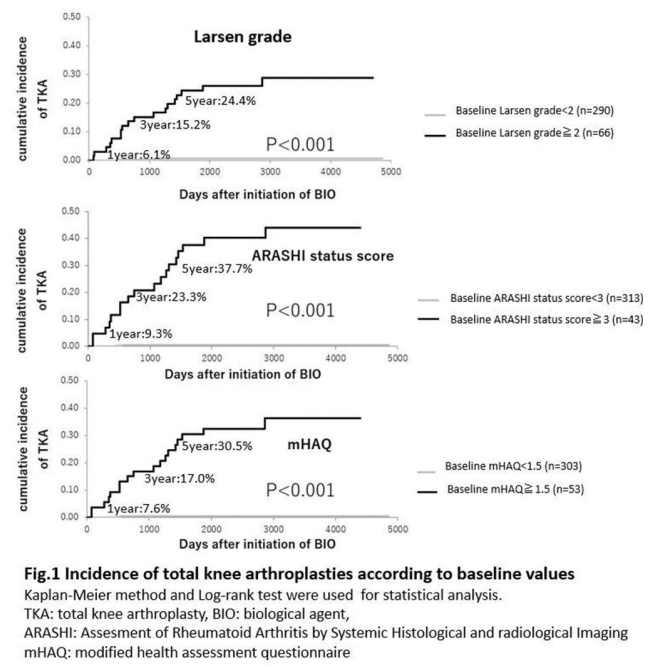

Disclosure of Interests: None declared DOI: 10.1136/annrheumdis-2019-eular.5325 